University of San Diego

Digital USD

Spring 5-23-2020

\title{
The Supplemental Impact of Telephone-Based Lifestyle Modification Counseling in an Outpatient Weight Loss Clinic
}

Leslie Rangel Gonzalez BSN, RN

University of San Diego

Karen Macauley PhD, DNP, FNP-BC, GNP-BC

University of San Diego

Tony Rosales DNP, RN, CNS, FNP-BC

University of San Diego

Follow this and additional works at: https://digital.sandiego.edu/dnp

Part of the Nursing Commons

\section{Digital USD Citation}

Rangel Gonzalez, Leslie BSN, RN; Macauley, Karen PhD, DNP, FNP-BC, GNP-BC; and Rosales, Tony DNP, RN, CNS, FNP-BC, "The Supplemental Impact of Telephone-Based Lifestyle Modification Counseling in an Outpatient Weight Loss Clinic" (2020). Doctor of Nursing Practice Final Manuscripts. 117.

https://digital.sandiego.edu/dnp/117

This Doctor of Nursing Practice Final Manuscript is brought to you for free and open access by the Theses and Dissertations at Digital USD. It has been accepted for inclusion in Doctor of Nursing Practice Final Manuscripts by an authorized administrator of Digital USD. For more information, please contact digital@sandiego.edu. 


\section{Final Manuscript}

The Supplemental Impact of Telephone-Based Lifestyle Modification Counseling in an Outpatient Weight Loss Clinic

Leslie Rangel Gonzalez, BSN, RN

University of San Diego

Karen Macauley, PhD, DNP, FNP-BC, GNP-BC

Tony Rosales, DNP, RN, CNS, FNP-BC 


\begin{abstract}
Purpose: The purpose of the project is to implement effective, convenient, and efficient weight loss counseling in today's fast-paced mobile society. The project will reinforce current physical activity guidelines in correlation with the clinic's standard dietary guidelines and pharmacological interventions. Patient adherence and motivation will be supported through lifestyle modification counseling via telephone.

Background: The prevalence of obese adults in the United States (US) increased from 33.7 percent in 2007-2008 to 39.6 percent in 2015-2016. Abnormal or excess weight gain may further impair health, as it correlates with the development of multiple preventable comorbidities such as cardiovascular disease, diabetes mellitus, and some cancers such as breast cancer or colon cancer. Overweight and obese patient's pose a significant health care burden. Total costs attributable to the obese and overweight population is predicted to double every decade costing about $\$ 860.7$ - $\$ 956.9$ billion by 2030, accounting for 16-18 percent of total US health care costs (Mobley \& Baum, 2015). According to the CDC, (2018), the prevalence of obesity accounts for more than one-third (36.5 percent) of overweight and obese adults in the US, and further claims that in 2008 the estimated annual medical cost in the US attributed to obesity was \$147 billion.
\end{abstract}

Practice Change: Three mandatory telephonic reminders were added to each patient's weight loss plan on day three, day seven, and week six of the program.

Outcomes: Implementation of telephone-based intensive lifestyle modification counseling as an evidence-based intervention is a valuable method to improve weight loss outcomes, enhance clinical efficiency, maintain safety, and increase patient satisfaction. 
Conclusion: Early detection, diagnosis, and management of overweight or obese patients are vital to prevent morbidity, mortality, improve quality of life, and prolong life expectancy without suffering. Evidence-based interventions are key to help facilitate healthy weight loss goals and reduce both patient and health care system costs. 


\section{The Supplemental Impact of Telephone-Based Lifestyle Modification Counseling in an Outpatient Weight Loss Clinic}

\section{Background}

Being obese or overweight is becoming the new norm in today's American society. Approximately 66 percent of Americans in 2009-2014 were considered overweight or obese, compared with 62 percent in 2004, and 55 percent in 1994 (Hruby \& Hu, 2015). Carrying excess weight in the human body can be detrimental, as it increases the risk of many chronic diseases exponentially in both children and adults over time (Hruby \& Hu, 2015). It is well known that excessive weight gain is a primary determinant to morbidity and mortality in the United States (US) (Hales, Fryar, Carroll, Freedman, \& Ogden, 2018). Furthermore, obesity has become a significant public health crisis. The prevalence of US adults with obesity has increased from 33.7 percent in 2007-2008 to 39.6 percent in 2015-2016 (Hales, Fryar, Carroll, Freedman, \& Ogden, 2018). Abnormal weight gain may further impair health, as it correlates with the development of multiple preventable chronic comorbidities which are currently managed in today's health care system and impedes activities of daily living (Mobley \& Baum, 2015). Heart disease and cancer are the number one and two causes of death in the US. Obesity is a leading contributing cause of both conditions, along with other related health problems including arthritis, high blood pressure, high cholesterol, diabetes mellitus, gallbladder disease, and sleep disorders (Mobley \& Baum, 2015).

Additionally, patients who are overweight or obese pose a significant health care burden. Total health care costs, attributable to the obese and overweight population, are predicted to double every decade, costing about $\$ 860.7$ - $\$ 956.9$ billion by 2030 , accounting for $16-18$ percent of total US health care costs (Mobley \& Baum, 2015). According to the CDC (2018), the 
prevalence of obesity accounts for more than one-third (36.5 percent) of overweight adults in the US with estimated annual medical costs of $\$ 147$ billion in 2008. Furthermore, Americans spent approximately $\$ 480.7$ billion in direct health care costs in 2016 on conditions related to risk factors of obesity and overweight. Lost economic productivity was calculated at \$1.24 trillion (Kelly., 2018), Obesity is a health concern in the US and worldwide (Mobley \& Baum, 2015). The World Health Organization (WHO) considers a person with a Body Mass Index (BMI) of greater than or equal to 25 to be overweight, and a person with a BMI of 30 or higher is considered to be obese (Flegal, Carroll, Ogden, \& Curtin, 2010).

\section{Evidence for Problem}

Obesity is a global health concern that has more than doubled since 1980 (WHO, 2014). In 2016, 1.9 billion adults were overweight, and 500 million were obese (WHO, n.d.). A calculated loss of economic productivity was estimated at $\$ 1.24$ trillion (Kelly, 2018). People continue to struggle to eat healthy for many reasons, such as economic factors, lack of education, and a busy work lifestyle. As a result, weight gain and obesity are a huge concern and many people find it difficult to eat healthy given the available choices. Successful weight loss strategies need to be thoroughly discussed and addressed by both clinicians and patients to improve clinical practices and adopt new standards. Education is a crucial component to weight loss management and clinicians are uniquely positioned to educate patient on proper diet and exercise. Overall, a healthy, well-proportioned diet plan along with exercise may improve patient outcomes and reduce excessive weight gain and obesity (Healthy People 2020 n.d.).

Weight management awareness and action against this preventable condition needs to be prioritized and addressed at every doctor's visit. According to the CDC (2013), approximately 33 percent of adults are overweight, and 26 percent are obese. At the study site (an outpatient 
weight loss clinic), stakeholders and clients voiced desire for adding one-on-one diet and exercise counseling to the treatment plan. This individual lifestyle modification counseling was not standard practice at this clinic. Therefore, this evidence-based practice project (EBP) focused on implementing attainable and sustainable weight management techniques to promote weight loss.

\section{Evidence-Based Intervention}

The implementation of modifiable factors such as physical activity and telephone-based counseling was a new intervention provided at the clinic for overweight and obese patients to help assist during their weight loss management. Telephone-based counseling can provide coaching opportunities for reinforcement and adherence of physical activity and the clinic's existing dietary recommendations (low glycemic diet) to achieve weight loss and reduce BMI within a six-week time period.

According to the American Heart Association, (2018), adults should be exercising at least 150 minutes a week for weight maintenance, of moderate-intensity aerobic activity (at least brisk walking) to help maintain weight loss. The article further advises that at least 300 minutes of moderate to high-intensity aerobic exercise (at least brisk walking) is necessary for weight reduction.

Nutritional counseling that encourages a diet in which the glycemic level is controlled to reduce $\mathrm{BMI}$ is an effective intervention in the management of overweight and obesity (Larsen et al., 2010). According to Larsen et al., (2010), a diet with a low glycemic index plays a vital role in stimulating the loss of weight in individuals who are overweight or obese. The designation of low glycemic index food was initially meant for diabetic patients to help stabilize the level of glucose in the blood. Food with a low glycemic index represents a diet that limits the secretion 
of insulin from the pancreatic cells by preventing fluctuations in blood glucose. Since insulin encourages glucose uptake by the body's cells, a reduction in insulin level promotes the utilization of fats for the generation of energy (Larsen et al., 2010).

In a randomized trial study by Biese et al., (2014)., telephone-based counseling and callbacks were implemented in an outpatient setting to promote competence and compliance with the patient's outpatient instructions. The results showed a significant increase in patient satisfaction along with successful outcomes involving medication compliance of the medication regimen, patient adherence to instructions, and decreased readmissions. Furthermore, calls were provided on days 1-3, then again on days 5-8, and lastly 30-35 days (Biese et al., 2014). High patient and provider satisfaction were found with the primary care providers who were fully involved and engaged in their patient's weight loss management journey using lifestyle modification counseling (Bennett et al., 2015).

A systematic review revealed how face-to-face counseling or via telephone with patients in the primary care setting showed promise in improving successful weight loss outcomes (Wadden et al., 2014). Phone calls were made, and weight loss counseling was provided (diet and physical activity), measurements were obtained, and a reduction in their body mass was achieved. The article further mentions not only may it promote initial weight loss but also longterm weight maintenance, primarily when guided by an interventionist (e.g., medical assistant, registered nurse, registered dietitians) in overweight/obese patients (Wadden et al., 2014). Another meta-analysis by Johns et al., (2014), provided promising weight loss evidence when it comes to implementing both diet and exercise in a counseling format. This technique has not only shown to be beneficial in weight reduction but also in keeping the weight off (Johns et al., 
2014). Guidance, support, and encouragement during this vulnerable moment have shown to be a successful complement to meet one's ideal body weight (Johns et al., 2014).

According to O'Hara et al., (2013), upon completion of the study, results were reviewed, and the findings showed telephone-based counseling was a contributory factor to improved patient outcomes, and sustained weight loss. Increased physical activity and nutritional changes, undertaken through telephone-based counseling method showed to be effective. Furthermore, O'Hara et al., (2017), in a quasi-experimental study, showed that those with goal setting weight management involving physical activity was effective in achieving weight loss, whereas eating more fruits and vegetables resulted in no negative or positive difference in weight. Overall, the study showed that collectively lifestyle modification of physical activity and nutrition via telephone-based counseling intervention had a positive impact on weight loss.

\section{Benchmark}

Outcome indicators consist of monitoring weight in pounds and BMI over a six-week period of time for participants desiring weight loss at a weight loss clinic. By the end of Week 6 , the project will seek to achieve a reduction of BMI between 3-5 percent, and weight reduction between 2-10 pounds.

\section{Evidence Based Practice Question (PICO Question)}

Will the implementation of telephone-based counseling (diet and exercise), for adults seeking outpatient weight loss services, result in a reduction of BMI by 3-5 percent over a sixweek period?

\section{Project Plan Process}


The intent of this EBP project was to implement telephone-based counseling of diet and physical activity, and the clinic's current diet preference of a low-glycemic diet to achieve weight loss within six weeks. Once stakeholder and IRB approval was obtained, a provider and staff mobile in-service (telephone-based counseling) on the proposed intervention was conducted. Then face to face patient-provider office visits were arranged. During the initial visit, the provider reviewed the weight loss program with the patient. The program included diet, exercise, pharmacological interventions if necessary, and telephonic follow-up calls. On the same day, baseline body measurements (weight and BMI) were obtained. The same body measurements were then obtained for every follow-up visit on day seven, day 30, and on week six. Phone calls were conducted to counsel patients on day three after the initial visit, day seven, and on day 30 . Physical activity of 300 minutes of at least brisk walking was recommended weekly to lose weight. Once the target weight was reached, physical activity was lessened to 150 minutes a week of at least continued brisk walking to maintain the target weight. Electronic medical record (EMR) reminders were used to alert ancillary staff to collect data during each patient visit. The program guide each patient throughout their weight loss journey and reinforced the importance of following the weight loss plan. The counseling via telephone was used to encourage, motivate, inspire, and assist in accomplishing their weight loss goals and maintain healthy body weight.

\section{Evaluation Results}

Six months of pre-data (body weight and BMI) were collected from chart reviews of 13 individuals presenting to the clinic for weight loss to determine percent weight loss over 6 weeks. The pre-intervention group included 11 females, two males, and ages ranged from 25-58 years old, all of who were either overweight or obese. The intervention group was composed of 13 individuals, 11 females, two males, all ranging anywhere from 23-55 years old, and were 
either overweight or obese seeking to lose weight. The intervention group's body weight and BMI measurements were collected on day seven, day 30 , and on week six, to determine percent weight loss over a six-week time period.

The line graph in figure 1 compares the percentage of weight loss over the course of sixweeks for the pre-intervention and the intervention group. The results show a greater BMI reduction in the intervention group, which met the project benchmark of 3-5 percent. The preintervention group on average lost a total of 2 percent and the intervention group lost on average just over 4 percent of their body weight.

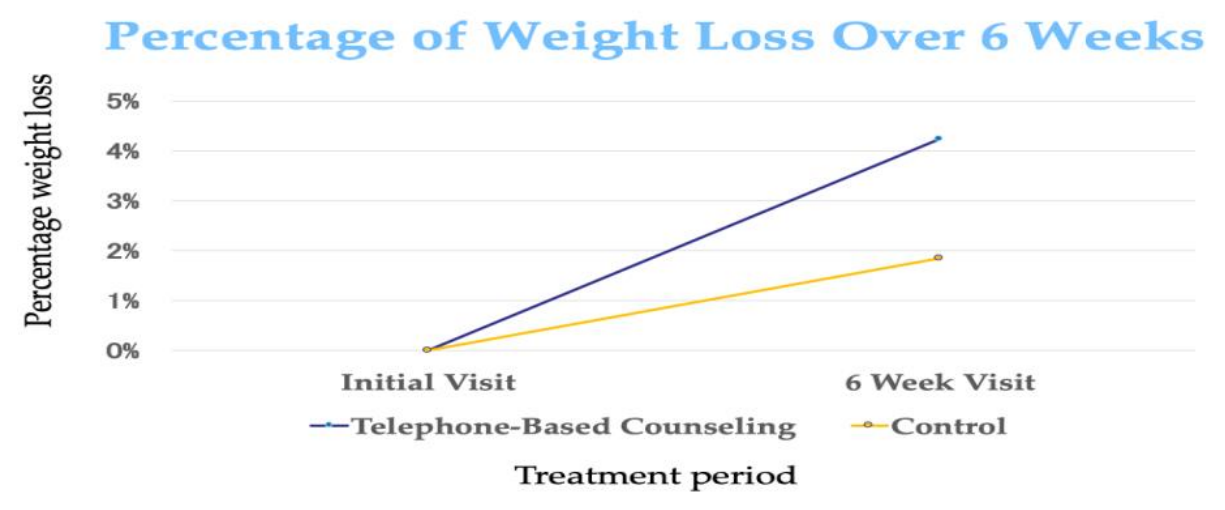

Figure 1. Six-week pre/post weight loss average.

\section{Implications for Clinical Practice}

Based on the results obtained at the completion of the project, weight reduction was achieved by reiterating, reinforcing, and motivating each patient through the process via telephone. The use of this technology was more convenient to both the ancillary staff and patients. Patients could receive encouraging calls that fit more realistically with their daily schedules. This method allowed patients to feel more comfortable to speak freely with the telephone-based counseling calls about any struggles they may be encountering through their weight loss journey. Anecdotally, a rapport and connection with each patient was achieved during each call. Patients vocalized the counseling sessions made them feel truly cared for and 
how the telephone calls helped motivated them to stay on track. Additionally, a callback number during business hours was provided for any questions or clarifications they may have, or if they felt discouraged. This allowed patients to express their personal situations, such as not seeing the pounds drop immediately or work-related issues. They expressed how having a professional to call and voice their concerns gave them the boost they needed when they needed it the most. Although the project was successful, there were some challenges. Such as, a small sample of patients enrolled in the study. Patients would get discouraged if they were not losing weight fast enough, which resulted in some individuals not following up with their weight-in sessions, and not responding to follow-up phone calls. Lastly, some elements of the data entry were missed by the staff. Methods to help guide these pitfalls include, increasing the duration of the program from six-weeks to six months or even a year would allow for more data points, collecting a larger sample of patients, and scheduling calls to fit the patients and office's schedule.

Sustainability is the ultimate goal of this EBP project. The key is to achieve patient weight loss, sustain the weight loss, promote overall health, and improve quality of life in overweight and obese patients. Through successful completion of this project, essential data can be utilized to show how implementing telephone-base counseling sessions on weight loss to current standard of care can improve patient outcomes. The first step begins with initiating realistic outcomes and clinic protocols. Secondly, add reminders for the staff by using and assigning flags to the clinic's EMR. The EMR can be used as a successful tool for future and upcoming phases of implementation and assist in guiding new or upcoming tasks that may need to be completed. 


\section{Cost/Benefit Analysis}

Developing and applying this EBP project into practice is affordable and cost-effective. Additional costs were not incurred as the current office staff could absorb the telephone followup counseling sessions. Continued efforts to reduce excessive weight gain in our community will aid in reducing long-term health care cost. Current health care dollars are spent due to secondary conditions or sequelae attributable to excess weight, this is far costlier than the implementation of this EBP project.

Abnormal weight gain and obesity are related to conditions such as hypertension, prediabetes, type II diabetes, and cardiovascular disease to name a few, which all tend to be quite costly (Yang \& Colditz, 2015). The yearly cost of managing and treating diabetes alone is estimated at more than 35 billion dollars (CDC, 2014). The total estimated cost of diagnosed diabetes mellitus in the United States in 2012 was $\$ 245$ billion in both direct and indirect costs of health damages; one in three Medicare dollars is currently spent on people with diabetes mellitus (CDC, 2014). Obesity is the leading cause of type II diabetes and it is a preventable condition. Preventing obesity is a very cost-effective method to reduce the financial burden on our health care system and on the individual.

The project provides a cost-effective approach to mitigating the current financial burden caused by abnormal weight gain and excessive weight gain of patients. For providers, this project can be instrumental in effectively reducing patient weight and aid in sustaining healthy weight loss for their patients in a cost-effective manner.

\section{Conclusions}

Overweight and obesity are major current health care challenges. It is imperative that clinicians begin to address obesity in the population. More nutritional and behavioral forms of 
prevention are necessary to reduce common contributing risk factors that will improve overall health and increase the life span of obese or overweight patients.

Telephone-base counseling has shown successful outcomes when compared to individuals who did not receive one-on-one telephone-based counseling. Weight loss programs should consider implementing reinforcement, reiteration, and encouraging telephone-based counseling. This method will assist in guiding patients through their weight loss process and providing encouragement improves the progress towards meeting weight loss goals/ideal body weight. Telephone-based counseling is an effective compliment to concurrent treatments and increases adherence via telephone, guidance, support through patient's weight loss journey, and provides opportunities for patients to ask questions. Additionally, this introduces longer-term weight loss strategies to help patients sustain their weight loss. Applying telephone-based counseling of both diet and physical activity to clinical practice positively affects long-term adjustments and promotes positive outcomes to help resolve the obesity health crisis and improve longevity. 


\section{References}

American Heart Association Recommendations for Physical Activity in Adults and Kids. (2018).

Retrieved April 3, 2019, from www.heart.org website: https://www.heart.org/en/healthyliving/fitness/fitness-basics/aha-recs-for-physical-activity-in-adults

Bennett, W. L., Wang, N. Y., Gudzune, K. A., Dalcin, A. T., Bleich, S. N., Appel, L. J., \& Clark, J. M. (2015). Satisfaction with primary care provider involvement is associated with greater weight loss: results from the practice-based POWER trial. Patient education and counseling, 98(9), 1099-1105.

Biese, K., LaMantia, M., Shofer, F., McCall, B., Roberts, E., Stearns, S. C., \& Busby-Whitehead, J. (2014). A randomized trial exploring the effect of a telephone call follow-up on care plan compliance among older adults discharged home from the emergency department. Academic Emergency Medicine, 21(2), 188-195.

Centers for Disease Control and Prevention. (2018). Adult obesity facts $\mid$ Overweight \& Obesity. Retrieved on March 26, 2019 from https://www.cdc.gov/obesity/data/adult.html

Centers for Disease Control and Prevention, (2014). California surveillance data.

Retrieved from http://apps.nccd.cdc.gov/DDTSTRS/StatePage.aspx?state=California

Centers for Disease Control and Prevention. (2013). Community profile - County of San Diego, CA-Communities putting prevention to work. Retrieved March 23, 2019, from https://www.cdc.gov/nccdphp/dch/programs/communitiesputtingpreventiontowork/comm unities/profiles/obesity-ca_sandiego-county.htm

Flegal, K. M., Carroll, M. D., Ogden, C. L., \& Curtin, L. R. (2010). Prevalence and trends in obesity among US adults, 1999-2008. Jama, 303(3), 235-241. 
Hales, C. M., Fryar, C. D., Carroll, M. D., Freedman, D. S., \& Ogden, C. L. (2018). Trends in obesity and severe obesity prevalence in US youth and adults by sex and age, 2007-2008 to 2015-2016. Jama, 319(16), 1723-1725.

Hruby, A., \& Hu, F. B. (2015). The Epidemiology of Obesity: A Big Picture. PharmacoEconomics, 33(7), 673-689. https://doi.org/10.1007/s40273-014-0243-x

Johns, D. J., Hartmann-Boyce, J., Jebb, S. A., Aveyard, P., \& Group, B. W. M. R. (2014). Diet or exercise interventions vs combined behavioral weight management programs: a systematic review and meta-analysis of direct comparisons. Journal of the Academy of Nutrition and Dietetics, 114(10), 1557-1568.

Kelly L (2018). Obesity epidemic costs U.S. economy $\$ 1.72$ trillion. Washingtontimes

Larsen, T. M., Dalskov, S. M., van Baak, M., Jebb, S. A., Papadaki, A., Pfeiffer, A. F., ... \& Stender, S. (2010). Diets with high or low protein content and glycemic index for weightloss maintenance. New England Journal of Medicine, 363(22), 2102-2113.

Mobley, D., \& Baum, N. (2015). The Obesity Epidemic and Its Impact on Urologic Care. Reviews in Urology, 17(3), 165-170.

Nutrition, Physical Activity, and Obesity | Healthy People 2020. (n.d.). Retrieved January 15, 2020, from https://www.healthypeople.gov/2020/leading-health-indicators/2020-lhitopics/Nutrition-Physical-Activity-and-Obesity/data

O’Hara, B. J., Phongsavan, P., Eakin, E. G., Develin, E., Smith, J., Greenaway, M., \& Bauman, A. E. (2013). Effectiveness of Australia's Get Healthy Information and Coaching Service®: maintenance of self-reported anthropometric and behavioural changes after program completion. BMC Public Health, 13(1), 175. 
O’Hara, B. J., Gale, J., McGill, B., Bauman, A., Hebden, L., Allman-Farinelli, M., ... \& Phongsavan, P. (2017). Weight-Related goal setting in a Telephone-Based preventive Health-Coaching program: demonstration of effectiveness. American Journal of Health Promotion, 31(6), 491-501.

Wadden, T. A., Butryn, M. L., Hong, P. S., \& Tsai, A. G. (2014). Behavioral treatment of obesity in patients encountered in primary care settings: a systematic review. Jama, 312(17), 1779-1791.

World Health Organization. (2014). Retrieved March 20, 2019, from http://www.who.int/en/ World Health Organization. (n.d.). Retrieved March 20, 2019, from http://www.who.int/en/ Yang, L., \& Colditz, G. A. (2015). Prevalence of overweight and obesity in the United States, 2007-2012. JAMA Internal Medicine, 175, 1412-1413. 